\title{
THE STRUCTURE OF THE EXPONENTS AND SYMMETRIES OF AN OPERATOR STABLE LAW
}

\author{
by \\ Mark M. Meerschaert ${ }^{1}$ \\ Albion College \\ Jerry Alan Veeh \\ Auburn University
}

31 October 1992

\begin{abstract}
Operator stable laws and generalized domains of attraction are the natural multidimensional analogues of the classical stable laws and domains of attraction in one variable. Exponents of operator stable laws generalize the index of a classical stable law. In this paper we present a series of decomposition theorems which completely characterize the algebraic structure of exponents and symmetries. We give an example of an operator stable law whose symmetry group is a one parameter subgroup, so that its commuting exponent is not unique. Our results illuminate the tail behavior of operator stable laws, which are governed by the exponents, and which must be preserved by the symmetries. We also discuss applications to generalized domains of attraction, including moments, centering constants, tails, and norming operators.
\end{abstract}

AMS 1980 Subject classification: 60F05

Key words and phrases: operator stable, random vector, exponent, symmetry.

Running head: OPERATOR STABLE LAWS

${ }^{1}$ Partially supported by National Science Foundation grants DMS-89-23068 and DMS-91-03131. 


\section{Introduction}

Suppose that $X, X_{1}, X_{2}, X_{3}, \ldots$ are independent random vectors on $\mathbf{R}^{d}$ with common distribution $\mu$, and that $Y$ is a random vector on $\mathbf{R}^{d}$ with distribution $\nu$. Suppose that $\nu$ is full, i.e. that it cannot be supported on a proper hyperplane. If there exist linear operators $A_{n}$ on $\mathbf{R}^{d}$ and constant vectors $b_{n} \in \mathbf{R}^{d}$ such that

$$
A_{n}\left(X_{1}+\cdots+X_{n}\right)-b_{n} \Rightarrow Y
$$

then we say that $\mu$ belongs to the generalized domain of attraction of $\nu$. In this case the distribution of the partial sum $\left(X_{1}+\cdots+X_{n}\right)$ can be usefully approximated by that of the normalized limit law $A_{n}^{-1}\left(Y+b_{n}\right)$ for $n$ large. Operator stable laws are those full probability measures on $\mathbf{R}^{d}$ which have a nonempty generalized domain of attraction.

The theory of operator stable probability measures was begun by Sharpe (1969). A full probability measure $\nu$ on $\mathbf{R}^{d}$ is said to be operator stable if there is a linear operator $B$ on $\mathbf{R}^{d}$ (called an exponent of $\nu$ ) and a vector valued function $b_{t}$ so that for all $t>0$

$$
\nu^{t}=t^{B} \nu * \delta\left(b_{t}\right) .
$$

Here $\nu$ is known to be infinitely divisible, so $\nu^{t}$, the $t$ th convolutory power, can be defined by the characteristic function. The operator $t^{B}$ is defined via power series as $\exp \{B \ln t\}$, and for any linear operator $A$ we define the measure $A \nu(E)=\nu\left(A^{-1} E\right)$. One of the central features of the theory of operator stable probability measures is the interplay between ideas from probability theory, linear algebra, and Lie theory. A consequence of this interplay is that some 'probabilistic' facts about operator stable probability measures are most easily established using algebraic arguments. In this paper we use algebraic techniques to prove some facts about the structure of exponents and symmetries of full operator stable probability measures.

Consider for the moment the case $d=1$. When (1.1) holds we say that $\mu$ belongs to the domain of attraction of the nondegenerate probability distribution $\nu$. The classical stable laws are those nondegenerate probability measures on $\mathbf{R}^{1}$ which have a nonempty domain of attraction. The exponent $B$ of a stable law is a unique real number in the interval $\left[\frac{1}{2}, \infty\right)$, and its reciprocal $\alpha$ is the classical index of the stable law. If $\alpha=2$ then $\nu$ is a nondegenerate normal law on $\mathbf{R}^{1}$. In the case $\alpha \neq 2$ the index governs the behavior of moments, tails, centering constants, and norming. The absolute moments $E|Y|^{\rho}$ are finite whenever $\rho<\frac{1}{\alpha}$, and infinite for $\rho \geq \frac{1}{\alpha}$. If $X$ is attracted to $Y$ then $E|X|^{\rho}$ is finite whenever $\rho<\frac{1}{\alpha}$, and infinite whenever

$\rho>\frac{1}{\alpha}$. The tail functions $P(|Y|>t)$ and $P(|X|>t)$ vary regularly with index $-\alpha$, and the norming constants $A_{n}$ form a regularly varying sequence with index $-\frac{1}{\alpha}$. The centering constants $b_{n}$ can be omitted when $\alpha<1$, and when $\alpha>1$ we can center to zero expectation. 
In general, operator stable probability measures do not have unique exponents. If $\mathcal{E}(\nu)$ denotes the collection of exponents of the operator stable measure $\nu$ and $\mathcal{S}(\nu)=\{A: A \nu=\nu * \delta(a)$ for some $a\}$ denotes the symmetry group of $\nu$, then Holmes, Hudson, and Mason (1982) established that

$$
\mathcal{E}(\nu)=B+T \mathcal{S}(\nu)
$$

where $B \in \mathcal{E}(\nu)$ and $T \mathcal{S}(\nu)$ is the tangent space (Lie algebra) of the compact group $\mathcal{S}(\nu)$. This result shows that the structure of the collection of exponents and the structure of the symmetry group are closely interrelated. Hudson, Jurek, and Veeh (1986) established the existence of an exponent $B_{0} \in \mathcal{E}(\nu)$ which commutes with every element of $\mathcal{S}(\nu)$. Such exponents are called commuting exponents and play an important role in our structure results.

Symmetries complicate the behavior of the norming operators $A_{n}$ for generalized domains of attraction. Take for example the case of a standard normal limit so that the symmetry group $\mathcal{S}(\nu)$ consists of every orthogonal linear operator on $\mathbf{R}^{d}$. If $E\|X\|^{2}$ is finite then we get convergence in (1.1) with $A_{n}=\left(\frac{1}{2}\right) I$ where $I$ denotes the identity operator on $\mathbf{R}^{d}$. But if $G_{n} \in$ $\mathcal{S}(\nu)$ is any sequence of symmetries, then we also get convergence with $A_{n}=$ $\left(\frac{1}{2}\right) G_{n}$. Michaliček (1972) showed that whenever (1.1) holds we have

$$
A_{[n t]} A_{n}^{-1} \rightarrow t^{-B} \mathcal{S}(\nu)
$$

for all $t>0$. Here the arrow indicates that the sequence on the left is limit point compact, and every limit point lies in the compact set on the right. Relation (1.4) is a regular variation condition on the sequence of norming operators $A_{n}$. It implies that the asymptotic behavior of $A_{n}$ will be like that of $n^{-B} G_{n}$ where $G_{n} \in \mathcal{S}(\nu)$. As in the one variable case, the exponent governs the behavior of norming operators. In the present case, however, there is an added complication due to the possible influence of multiple symmetries. The structure theorems presented in this paper were motivated by the authors' desire to understand the behavior of moments, tails, centering constants, and especially the norming operators for generalized domains of attraction. At the end of this paper we include some applications of our structure results, and indicate directions for future research.

\section{Preliminary Results}

We will begin by examining in more detail the properties of exponents. We recall that a linear operator on $\mathbf{R}^{d}$ is said to be semisimple if its minimal polynomial is the product of distinct prime factors. This is equivalent to the statement that the linear operator is diagonalizable over the complex numbers. A linear operator $N$ on $\mathbf{R}^{d}$ is nilpotent if $N^{k}=0$ for some $k$. One of the fundamental results of linear algebra is the $\mathrm{S}+\mathrm{N}$ Decomposition Theorem which states that if $T$ is a linear operator on $\mathbf{R}^{d}$, then $T$ has a 
unique representation as $T=S+N$ where $S$ is semisimple, $N$ is nilpotent, and $S N=N S$. Moreover, both $S$ and $N$ are polynomials in $T$. A proof of this result can be found in Hoffman and Kunze (1961). When we speak of the 'nilpotent part' or the 'semisimple part' of a linear operator, we are referring to the nilpotent or semisimple operator in the $S+N$ decomposition of the given operator. This leads to our first theorem.

Theorem 1. The nilpotent part of every $B \in \mathcal{E}(\nu)$ is the same, and it commutes with every element of the symmetry group $\mathcal{S}(\nu)$.

Proof: Choose a commuting exponent $B_{0}$ and write $B_{0}=S_{0}+N_{0}$ where $S_{0}$ is semisimple and $N_{0}$ is nilpotent. Now both $S_{0}$ and $N_{0}$ are polynomials in $B_{0}$. Hence $A S_{0}=S_{0} A$ and $A N_{0}=N_{0} A$ for every $A \in \mathcal{S}(\nu)$. To conclude the proof, it is enough to show that every exponent $B$ has the same nilpotent part $N_{0}$. Let $B \in \mathcal{E}(\nu)$ be arbitrary and write $B=S+N$ as before. Since $\mathcal{E}(\nu)=B_{0}+T \mathcal{S}(\nu)$, we may write $B=B_{0}+X$ where $X \in T \mathcal{S}(\nu)$. Then $B=\left(S_{0}+X\right)+N_{0}$ and so by uniqueness of the $\mathrm{S}+\mathrm{N}$ decomposition it will suffice to show that $S_{0}+X$ is semisimple. Since $S_{0}$ commutes with all $A \in \mathcal{S}(\nu)$ it also commutes with $X \in T \mathcal{S}(\nu)$. Now Billingsley (1966) showed that $\mathcal{S}(\nu)$ is conjugate to a subgroup of the orthogonal group. It follows that $T \mathcal{S}(\nu)$ is conjugate to a subspace of the space of skew symmetric operators. Thus every $X \in T \mathcal{S}(\nu)$ is of the form $W^{-1} Q W$ where $Q$ is skew symmetric, and so $X$ is semisimple. Since the sum of two semisimple operators which commute must also be semisimple, the theorem is proved. IThe proof of this theorem contains the germ of an interesting corollary.

Corollary. The set $\{\operatorname{Re}(\lambda): \lambda \in \sigma(B)\}$ (called the real spectrum of $B$ ) is independent of $B \in \mathcal{E}(\nu)$.

Proof: To see this suppose $B$ is a commuting exponent and $B+X, X \in$ $T \mathcal{S}(\nu)$, is any other exponent. Since $X$ is semisimple and $B X=X B$ we can choose a basis for $\mathbf{C}^{d}$ in which $X$ is diagonal and $B$ has Jordan form. The result follows from the fact that $X$, being conjugate to a skew operator, has purely imaginary eigenvalues.

Another useful result from linear algebra is the primary decomposition theorem. Suppose $A$ is a linear operator on $\mathbf{R}^{d}$. Factor the minimal polynomial of $A$ into powers of distinct primes as $p_{1}(x)^{e_{1}} \cdots p_{s}(x)^{e_{s}}$. By letting $U_{i}=\operatorname{ker} p_{i}(A)^{e_{i}}$ we obtain a direct sum decomposition $\mathbf{R}^{d}=U_{1} \oplus \cdots \oplus U_{s}$ where $U_{1}, \cdots, U_{s}$ are all $A$-invariant subspaces. This is called the primary decomposition theorem of linear algebra. (See Hoffman and Kunze (1961).) The projections $P_{1}, \ldots, P_{s}$ which correspond to this decomposition are polynomials in $A$. 
Theorem 2. Suppose $B_{0}$ is a commuting exponent of $\nu$ and $\mathbf{R}^{d}=U_{1} \oplus$ $\cdots \oplus U_{s}$ is the primary decomposition for $B_{0}$. Then each of the subspaces $U_{1}, \ldots, U_{s}$ is an invariant subspace for any $B \in \mathcal{E}(\nu)$ and any $A \in \mathcal{S}(\nu)$.

Proof: Let $S$ be the semisimple part of $B_{0}$. Since $S$ is a polynomial in $B_{0}$ we know that $S$ commutes with every exponent and every symmetry of $\nu$. Any linear operator which commutes with $S$ must preserve the eigenspaces of $S$, real or complex. Each $U_{i}$ represents either a real eigenspace of $S$, or the real part of the direct sum of the two complex eigenspaces of $S$ corresponding to a single complex conjugate pair of eigenvalues. So in either case, $U_{i}$ must be an invariant subspace for any exponent or symmetry.

Let us say that a linear operator is primary if its minimal polynomial is the power of a single prime polynomial. We will say that an operator stable law $\nu$ is primary if it has a primary commuting exponent. The theorem just proved reduces the problem of characterizing exponents and symmetries to the primary case. For $B$ commuting, each component $B_{i}=B P_{i}=P_{i} B$ is a primary commuting exponent of $\nu_{i}=P_{i} \nu$ and

$$
\begin{gathered}
\mathcal{E}(\nu) \subseteq \mathcal{E}\left(\nu_{1}\right) \oplus \cdots \oplus \mathcal{E}\left(\nu_{s}\right) \\
\mathcal{S}(\nu) \subseteq \mathcal{S}\left(\nu_{1}\right) \oplus \cdots \oplus \mathcal{S}\left(\nu_{s}\right) .
\end{gathered}
$$

Meerschaert (1991) contains an example due to J. Veeh showing that there may be strict inclusion, and moreover the primary components $\nu_{1} \cdots \nu_{s}$ need not be independent.

We may also obtain information about the sum of certain primary projections. Suppose $a_{1}, \ldots, a_{n}$ is an enumeration of the real spectrum of some (any) $B \in \mathcal{E}(\nu)$. Denote by $\pi_{i}=\sum_{\operatorname{Re}\left(\lambda_{j}\right)=a_{i}} P_{j}$ where $P_{1}, \ldots, P_{s}$ are the primary projections of $B$. Then $\pi_{i}$ does not depend on the choice of $B$ used to construct it. Moreover, each of the projections $\pi_{i}$ is a polynomial in $B$. This decomposition according to the real spectrum of an arbitrary exponent is called the spectral decomposition. The strikingly simple proof just given shows the advantage of algebraic methods over the geometrical approach used in Meerschaert (1991). It also illustrates the utility of commuting exponents.

\section{Exponents and Symmetries}

Before presenting our main theorems we introduce a bit of terminology. A vector $x$ is said to be of order $k$ if $k=\min \left\{j: N^{j} x=0\right\}$. A subspace $W$ is said to be of order $k$ if every non-zero element of $W$ is of order $k$. We also make the following two observations. If $W$ is a subspace of order $k>1$ then $N W$ is a subspace of order $k-1$ and $\operatorname{dim}(N W)=\operatorname{dim}(W)$. If $W$ is an $\mathcal{S}(\nu)$-invariant subspace, then $N W$ is also $\mathcal{S}(\nu)$-invariant, since $N$ commutes with every element of $\mathcal{S}(\nu)$. 
Theorem 3. Suppose $\nu$ is operator stable and let $N$ be the nilpotent part of (any) exponent $B \in \mathcal{E}(\nu)$. Then there is a direct sum decomposition $\mathbf{R}^{d}=V_{1} \oplus \ldots \oplus V_{n}$ so that

(i) The subspace $V_{j}$ is $\mathcal{S}(\nu)$-invariant and of order $j$

(ii) $V_{j}=N V_{j+1} \oplus W_{j}$ where $W_{j}$ is also $\mathcal{S}(\nu)$-invariant and of order $j$.

(iii) There is a basis that respects the direct sum decomposition of $(i)$ and (ii), in which every $A \in \mathcal{S}(\nu)$ has the form

$$
\left(\begin{array}{lll}
A_{1} & & \\
& \ddots & \\
& & A_{n}
\end{array}\right)
$$

where $A_{j}$ is the $\operatorname{dim}\left(V_{j}\right) \times \operatorname{dim}\left(V_{j}\right)$ square orthogonal matrix which represents the restriction of $A$ to $V_{j}$ and

$$
A_{j}=\left(\begin{array}{cc}
A_{j+1} & 0 \\
0 & C_{j}
\end{array}\right)
$$

where $C_{j}$ is a $\operatorname{dim}\left(W_{j}\right) \times \operatorname{dim}\left(W_{j}\right)$ square orthogonal matrix.

Proof: First suppose that $\mathcal{S}(\nu)$ is a subgroup of the orthogonal group. Let $K_{j}=\operatorname{ker} N^{j}$, and observe that since $N$ commutes with all elements of the symmetry group each of the subspaces $K_{j}$ is $\mathcal{S}(\nu)$-invariant. Furthermore, since the symmetry group is contained in the orthogonal group, the perp space of any $\mathcal{S}(\nu)$-invariant subspace is also $\mathcal{S}(\nu)$-invariant. Assume $N^{n}=$ 0 and set $V_{n}=K_{n-1}^{\perp}$ and also set $W_{j}=\left(N V_{j+1} \oplus K_{j-1}\right)^{\perp} \cap K_{j}$. It is straightforward to use the above comments to see that these subspaces are $\mathcal{S}(\nu)$-invariant and of the asserted order.

Now let $\left\{b_{i n}: 1 \leq i \leq \operatorname{dim}\left(V_{n}\right)\right\}$ be an arbitrary orthonormal basis for $V_{n}$. For $j<n$ define $b_{i j}=N b_{i, j+1}, 1 \leq i \leq \operatorname{dim}\left(V_{j+1}\right)$. If $\operatorname{dim}\left(V_{j}\right)>$ $\operatorname{dim}\left(V_{j+1}\right)$, complete the basis for $V_{j}$ by letting $\left\{b_{i j}: \operatorname{dim}\left(V_{j+1}\right)+1 \leq i \leq\right.$ $\left.\operatorname{dim}\left(V_{j}\right)\right\}$ be an arbitrary orthonormal basis for $W_{j}$. Each $V_{j}$ is $A$-invariant, and we may write

$$
A b_{i j}=\sum_{k=1}^{\operatorname{dim}\left(V_{j}\right)} a_{k i}^{j} b_{k j} .
$$

Also

$$
A N b_{i, j+1}=A b_{i j}=\sum_{k=1}^{\operatorname{dim}\left(V_{j}\right)} a_{k i}^{j} b_{k j}
$$


while

$$
\begin{aligned}
N A b_{i, j+1} & =N\left(\sum_{k=1}^{\operatorname{dim}\left(V_{j+1}\right)} a_{k i}^{j+1} b_{k, j+1}\right) \\
& =\sum_{k=1}^{\operatorname{dim}\left(V_{j+1}\right)} a_{k i}^{j+1} N b_{k, j+1} \\
& =\sum_{k=1}^{\operatorname{dim}\left(V_{j+1}\right)} a_{k i}^{j+1} b_{k j} .
\end{aligned}
$$

Using the fact that $N A=A N$ we find that $a_{k i}^{j+1}=a_{k i}^{j}$ for all $1 \leq i, k \leq$ $\operatorname{dim}\left(V_{j+1}\right)$. This means that $A_{j}=A_{j+1}$ on $N V_{j+1}$, as asserted.

Now suppose $\mathcal{S}(\nu)$ is not a subgroup of the orthogonal group. For any invertible linear operator $T$ it is true that $\mathcal{S}(T \nu)=T \mathcal{S}(\nu) T^{-1}$, and since $\mathcal{S}(\nu)$ is conjugate to a subgroup of the orthogonal group there is an operator $U$ so that the symmetry group $\mathcal{S}(U \nu)$ of the operator stable law $U \nu$ is a compact subgroup of the orthogonal group. Apply the above argument to show that there is a basis $\left\{b_{i j}\right\}$ in which every element of $\mathcal{S}(U \nu)$ has the desired form. For every $A \in \mathcal{S}(\nu)$ we have $U A U^{-1} \in \mathcal{S}(U \nu)$, and the matrix of $U A U^{-1}$ in the basis $\left\{b_{i j}\right\}$ is the same as the matrix of $A$ in the basis $\left\{U^{-1} b_{i j}\right\}$. This concludes the proof.

Remark 1. In the basis just constructed the nilpotent operator $N$ has matrix

$$
\left(\begin{array}{ccccccc}
0 & N_{1} & 0 & 0 & \ldots & 0 & 0 \\
0 & 0 & N_{2} & 0 & \ldots & 0 & 0 \\
\vdots & & & & & & \vdots \\
0 & \ldots & & & \ldots & 0 & N_{n} \\
0 & \ldots & & & \ldots & 0 & 0
\end{array}\right)
$$

where $N_{j}$ is a $\operatorname{dim}\left(V_{j}\right) \times \operatorname{dim}\left(V_{j+1}\right)$ matrix of the form $\left(\begin{array}{l}I \\ 0\end{array}\right)$.

We now present a result which shows how exponents and symmetries can be represented simultaneously in a form similar to that of the previous theorem.

Theorem 4. Suppose that $\nu$ is operator stable and that $B$ is a commuting exponent of $\nu$. Then there is a direct sum decomposition $\mathbf{R}^{d}=U_{1} \oplus \ldots \oplus U_{s}$ into subspaces invariant under $B, \mathcal{S}(\nu)$, and $N$, and a basis of $\mathbf{R}^{d}$ which respects this direct sum decomposition in which

(i) Every element of the symmetry group is block diagonal, with the ith block being of size $\operatorname{dim}\left(U_{i}\right) \times \operatorname{dim}\left(U_{i}\right)$ and itself having the block structure specified in Theorem 3

(ii) The nilpotent operator $N$ is block diagonal, with the ith block being of size $\operatorname{dim}\left(U_{i}\right) \times \operatorname{dim}\left(U_{i}\right)$ and itself having the structure specified above 
(iii) The semisimple part of the exponent $B$ is block diagonal, with the ith block being of size $\operatorname{dim}\left(U_{i}\right) \times \operatorname{dim}\left(U_{i}\right)$ and being either a multiple of the identity or block diagonal consisting of blocks of the form $\left(\begin{array}{cc}c & -d \\ d & c\end{array}\right)$.

Proof: As in the proof above it suffices to consider the case in which $\mathcal{S}(\nu)$ is a subgroup of the orthogonal group. The subspaces $\left\{U_{l}\right\}$ are the subspaces given in the primary decomposition theorem for $B$. Each of these spaces is invariant under $\mathcal{S}(\nu)$ as well as $N$ and $B$, so it suffices to consider the restriction of all operators to one of the subspaces $U_{l}$. Denote by $S$ the semisimple part of the exponent $B$ restricted to $U_{l}$. We will be done if we show how to construct a basis in which it has the desired form. If $S$ has a single real eigenvalue $c$ we have $S=c I$ and we simply construct a basis of $U_{l}$ as in Theorem 3. Suppose then that $S$ has a single complex conjugate pair of eigenvalues $c \pm i d$. Complexify so that now $S$ is diagonalizable and every $A \in \mathcal{S}(\nu)$ is unitary. We can write the complexification of $U_{l}$ as $\mathcal{U}_{1} \oplus \mathcal{U}_{2}$ where $\mathcal{U}_{1}$ and $\mathcal{U}_{2}$ are the eigenspaces of $S$ belonging to $c+i d$ and $c-i d$ respectively. We note that $\mathcal{U}_{1}, \mathcal{U}_{2}$ are $\mathcal{S}(\nu)$-invariant. Write $S=S_{1} \oplus S_{2}$ and $A=A_{1} \oplus A_{2}$ using this direct sum decomposition. Now use the construction of the previous theorem to obtain a basis $\left\{b_{i j}\right\}$ for $\mathcal{U}_{1}$ in which the conclusions of that theorem hold for any $A_{1}$. All of the arguments extend to complex vector spaces without difficulty. If $S b=(c+i d) b$ then $S \bar{b}=(c-i d) \bar{b}$ and so $\left\{\bar{b}_{i j}\right\}$ forms a basis for $\mathcal{U}_{2}$. Write $A b_{i j}=\sum \delta_{k l} b_{k l}$ and $A \bar{b}_{i j}=\sum \gamma_{k l} \bar{b}_{k l}$. Since $A\left(b_{i j}+\bar{b}_{i j}\right)$ is real and hence equal to its own complex conjugate, we must have $\gamma_{k l}=\bar{\delta}_{k l}$ which means that $A_{2}=\bar{A}_{1}$. We will now show that $\left\{\operatorname{Re}\left(b_{i j}\right), \operatorname{Im}\left(b_{i j}\right)\right\}$ is a basis for $U_{l}$ in which the results of the previous theorem hold, and in addition the matrix of $B$ is block diagonal with blocks of the form $\left(\begin{array}{cc}c & -d \\ d & c\end{array}\right)$. We know that the matrix of $A$ in the basis $\left\{b_{11}, b_{21}, \ldots\right\}$ is of the form $\left(\begin{array}{ll}A_{1} & \\ & A_{2}\end{array}\right)$ where $A_{2}=\bar{A}_{1}$ and $A_{1}$ is unitary. Write $A_{1}=\left(a_{i j}\right)$ so that $A_{2}=\left(\bar{a}_{i j}\right)$. A change of basis will be done in two steps. First note that in the basis $\left\{b_{11}, \bar{b}_{11}, b_{21}, \bar{b}_{21}, \cdots\right\}$ the matrix of $A$ is of the form

$$
M=\left(\begin{array}{ccccc}
a_{11} & 0 & a_{12} & 0 \ldots & \\
0 & \bar{a}_{11} & 0 & \bar{a}_{12} & \\
a_{21} & 0 & a_{22} & 0 & \\
0 & \bar{a}_{21} & 0 & \bar{a}_{22} & \\
\vdots & & & & \ddots
\end{array}\right)
$$

and so in the basis $\left\{\operatorname{Re}\left(b_{11}\right), \operatorname{Im}\left(b_{11}\right), \cdots\right\}$ we see that $A$ has the matrix $M^{\prime}=$ $C^{-1} M C$ where $C$ is block-diagonal with blocks of the form $\left(\begin{array}{cc}1 / 2 & 1 / 2 i \\ 1 / 2 & -1 / 2 i\end{array}\right)$. 
So $C^{-1}$ is block-diagonal with blocks of the form $\left(\begin{array}{cc}1 & 1 \\ i & -i\end{array}\right)$ and we compute that

$$
M^{\prime}=\left(\begin{array}{ccccc}
\alpha_{11} & \beta_{11} & \alpha_{12} & \beta_{12} \ldots & \\
-\beta_{11} & \alpha_{11} & -\beta_{12} & \alpha_{12} & \\
\alpha_{21} & \beta_{21} & \alpha_{22} & \beta_{22} & \\
-\beta_{21} & \alpha_{21} & -\beta_{22} & \alpha_{22} & \\
\vdots & & & & \ddots .
\end{array}\right)
$$

Here we have let $a_{p q}=\alpha_{p q}+i \beta_{p q}$. We know that the complex inner product of the $i$ th and $j$ th column of $A_{1}$ is equal to one if $i=j$ and is zero otherwise, since $A_{1}$ is unitary. From the fact that the inner product of a column with itself is 1 , we see that every column of $M^{\prime}$ is a unit vector. By taking the real and imaginary parts of the inner product of the $i$ th and $j$ th column we also see that the columns of $M^{\prime}$ are orthogonal. Therefore $M^{\prime}$ is orthogonal. This completes the proof.

Remark 2. In the basis just constructed the tangent space of the symmetry group consists of skew matrices. This is because the tangent space of the orthogonal group consists of the skew symmetric matrices. It should also be noted that the skew symmetric matrices in the tangent space of the symmetry group inherit the special form given in Theorem 3 . This follows immediately from the definition of the derivative. In the basis above any exponent may be written in the form $D+Q+N$ where $D=\sum_{i=1}^{n} a_{i} \pi_{i}$ as discussed earlier. Here $D$ is diagonal and $Q$ is skew and has the same block form as a symmetry. Moreover $D$ is unique. The uniqueness of $D$ is part of the spectral decomposition discussed previously.

Remark 3. Let $j_{1}, \ldots, j_{p}$ be the subset of $j=1, \ldots, n$ for which $\operatorname{dim}\left(V_{j}\right)>$ $\operatorname{dim}\left(V_{j+1}\right)$ and define $d_{r}=\operatorname{dim}\left(V_{j_{r}}\right)-\operatorname{dim}\left(V_{j_{r}+1}\right)$. Then the theorem implies that $\mathcal{S}(\nu)$ is isomorphic to a compact subgroup of $O\left(\mathbf{R}^{d_{1}}\right) \times \cdots \times O\left(\mathbf{R}^{d_{p}}\right)$. The dimensions $d_{1}, \ldots, d_{p}$ are uniquely determined by the nilpotent operator $N$. Hirsch and Smale (1974) show that for every $N$ nilpotent on $\mathbf{R}^{d}$ there is a basis for $\mathbf{R}^{d}$ in which the matrix of $N$ has a block-diagonal form with blocks of the form

$$
\left(\begin{array}{ccc}
0 & & \\
1 & \ddots & \\
& \ddots & 0 \\
& & 1
\end{array}\right)
$$

The matrix above is called an elementary nilpotent block. If $N$ has elementary nilpotent blocks of sizes $s_{1}, \ldots, s_{q}$ where $s_{1}<s_{2}<\cdots<s_{q}$ then $d_{r}$ is the number of blocks of size $s_{r}$, for each $r=1, \ldots, q$. See Hirsch and Smale for a more general discussion of the relationship between $N, d_{r}$, and $\operatorname{dim}\left(V_{j}\right)$.

\section{An Example}


In this section we give an example of an operator stable law with multiple commuting exponents. In this case, the primary decomposition (2.1) may depend on the choice of exponent. We give an example in $\mathbf{R}^{4}$. This example makes use of the fact that an operator stable measure is infinitely divisible, and that the Lévy measure $M$ of an operator stable measure can be defined by the equation

$$
M(E)=\int_{S} \int_{0}^{\infty} \mathbf{1}_{E}\left(t^{B} x\right) t^{-2} d t d K(x)
$$

where $K$ is a Borel measure on a Borel set $S$ which is intersected exactly once by each orbit $\left\{t^{B} v: t>0\right\}$. For more details of this sort of construction, see Hudson, Jurek, and Veeh (1986). The measure $K$ is called the mixing measure.

We begin with some notation. Denote $v_{1}=e_{1}, v_{2}=e_{3}, v_{3}=\left(e_{1}+\right.$ $\left.e_{3}\right) / \sqrt{2}$, and $v_{4}=\left(e_{1}+e_{4}\right) / \sqrt{2}$ where $\left\{e_{1}, \ldots, e_{4}\right\}$ is the standard basis of $\mathbf{R}^{4}$. Denote $J=\left(\begin{array}{cc}0 & -1 \\ 1 & 0\end{array}\right), Q=$ block $\operatorname{diag}\{J, 2 J\}$, and define a one parameter group by $\left\{g_{\theta}=e^{\theta Q}: 0 \leq \theta \leq 2 \pi\right\}$. Define a Borel measure $K$ on the unit sphere by

$$
K(E)=\sum_{i=1}^{4} \int_{0}^{2 \pi} i \cdot \delta_{v_{i}}\left(g_{\theta} E\right) d \theta / 2 \pi
$$

and let $\phi$ be the Lévy measure with exponent $B=I$ and mixing measure $K$. Let $\nu$ be the operator stable law with Lévy representation $(0,0, \phi)$. We now argue that $\mathcal{S}(\nu)$ consists of only the operators $g_{\theta}$. It will follow from this that all commuting exponents are of the form $I+t Q$ where $t$ is real. Thus if $t=0$ there is one primary component, and otherwise there are two. To show that $\mathcal{S}(\nu)$ consists only of the operators $g_{\theta}$, we argue as follows. Denote by $V_{i}=\left\{t^{B} g_{\theta} v_{i}: t>0, \theta \in \mathbf{R}\right\}$ and by $C_{i}=\left\{g_{\theta} v_{i}: \theta \in \mathbf{R}\right\}$.

(1) We know that there is a positive definite $W$ so that $W \mathcal{S}(\nu) W^{-1}$ is a subgroup of the orthogonal group. It would be convenient for our purposes if we knew that all symmetries were orthogonal. The bulk of this example consists of showing that this is in fact the case. We begin by examining $W$. It is clear that $W$ is by no means unique. We shall first show that $W$ can be assumed to have a special structure. Since $g_{\theta}=e^{\theta Q}$ is a symmetry must have $W e^{t Q} W^{-1}$ orthogonal for all $t$. Hence $W e^{t Q} W^{-1} W^{-1} e^{-t Q} W=I$, or $e^{t Q} W^{-2}=W^{-2} e^{t Q}$ for all $t$. Writing this in block form and using the fact that $W$ is positive definite and that the only matrices which commute with $J$ are multiples of a rotation matrix, we see that $W$ is block diagonal. Moreover, the diagonal blocks are positive multiples of $I$. We may therefore assume that $W=$ block diag $(I, \alpha I)$ where $\alpha>0$. 
(2) Utilizing the previous comment, if $A \in \mathcal{S}(\nu)$ and the matrix of $A$ in the standard basis is (in block form) $A=\left(\begin{array}{ll}A_{11} & A_{12} \\ A_{21} & A_{22}\end{array}\right)$ then $A^{\prime}=$ $W A W^{-1}=\left(\begin{array}{cc}A_{11} & \frac{1}{\alpha} A_{12} \\ \alpha A_{21} & A_{22}\end{array}\right) \in \mathcal{S}(W \nu)$ and $A^{\prime}$ is orthogonal.

(3) We look now in detail at $K^{\prime}$, the mixing measure of $\phi^{\prime}=W \phi$, which is also supported on a subset of the unit sphere. Since $W$ commutes with $g_{\theta}$ and $B$ we see that $W V_{i}=\left\{t^{B} g_{\theta} W v_{i}: t>0, \theta \in \mathbf{R}\right\}$. Let $v_{i}^{\prime}=W v_{i} /\left\|W v_{i}\right\|$ and let $C_{i}^{\prime}=\left\{g_{\theta} v_{i}^{\prime}: \theta \in \mathbf{R}\right\}$. Note that

$$
v_{i}^{\prime}= \begin{cases}v_{1} & i=1 \\ v_{2} & i=2 \\ \frac{1}{\sqrt{1+\alpha^{2}}}\left(e_{1}+\alpha e_{i}\right) & i=3,4 .\end{cases}
$$

Note also that $\operatorname{supp}\left(K^{\prime}\right)=\bigcup_{i=1}^{4} C_{i}^{\prime}$. We now compute $K^{\prime}\left(C_{i}^{\prime}\right)$.

$$
\begin{aligned}
K^{\prime}\left(C_{i}^{\prime}\right) & =\phi^{\prime}\left\{t^{B} x: t \geq 1, x \in C_{i}^{\prime}\right\} \\
& =W \phi\left\{t^{B} x: t \geq 1, x \in C_{i}^{\prime}\right\} \\
& =\phi\left\{W^{-1} t^{B} x: t \geq 1, x \in C_{i}^{\prime}\right\} \\
& =\phi\left\{t^{B} x: t \geq 1, x \in W^{-1} C_{i}^{\prime}\right\} .
\end{aligned}
$$

Now simple computations show that

$$
W^{-1} C_{i}^{\prime}= \begin{cases}C_{1} & i=1 \\ \frac{1}{\alpha} C_{2} & i=2 \\ \sqrt{\frac{2}{1+\alpha^{2}}} C_{i} & i=3,4\end{cases}
$$

Hence, from the fact that $t^{B} \phi=t \cdot \phi$ for the Lévy measure of an operator stable measure, the fact that $B=I$, and the definition of $K$, we see that

$$
K^{\prime}\left(C_{i}^{\prime}\right)= \begin{cases}1 & i=1 \\ 2 \alpha & i=2 \\ i \sqrt{\frac{1+\alpha^{2}}{2}} & i=3,4\end{cases}
$$

(4) Since any symmetry $A^{\prime}$ of $W \nu$ commutes with $B$ and is orthogonal, it is also a symmetry of $K^{\prime}$. In particular, since supp $\left(K^{\prime}\right)$ is the disjoint union of the $C_{i}^{\prime}, A^{\prime}$ must map each $C_{i}^{\prime}$ into a $C_{i}^{\prime}$ having the same mass under $K^{\prime}$. Simple calculation shows that the $C_{i}^{\prime}$ have distinct masses except when $\alpha=1 / 2$. In this case, $C_{1}^{\prime}$ and $C_{2}^{\prime}$ have the same mass, and it is possible that $A^{\prime} C_{1}^{\prime}=C_{2}^{\prime}$. Our immediate goal is to show that this doesn't happen. Suppose $A^{\prime} C_{1}^{\prime}=C_{2}^{\prime}$. Then $\alpha=1 / 2$. Choose $\theta$ so that $A^{\prime \prime}=g_{\theta} A^{\prime}$ has the property $A^{\prime \prime} e_{1}=e_{3}$. 
Then $A^{\prime \prime}=\left(\begin{array}{cccc}0 & 0 & a & b \\ 0 & 0 & c & d \\ 1 & 0 & 0 & 0 \\ 0 & \pm 1 & 0 & 0\end{array}\right)$ by orthogonality. Now $A^{\prime \prime} C_{3}^{\prime}=C_{3}^{\prime}$ since both $A^{\prime}$ and $g_{\theta}$ have this property. Hence $A^{\prime \prime} v_{3}^{\prime} \in C_{3}^{\prime}$. But $A^{\prime \prime} v_{3}^{\prime}=A^{\prime \prime} \frac{1}{\sqrt{1+\alpha^{2}}}\left(e_{1}+\alpha e_{3}\right)=\frac{1}{\sqrt{1+\alpha^{2}}}\left(\begin{array}{c}\alpha a \\ \alpha c \\ 1 \\ 0\end{array}\right)$. For this to be in $C_{3}^{\prime}$ we need $c=0$ and $\alpha a= \pm 1$. But by orthogonality $a^{2}+c^{2}=1$, so $a= \pm 1$. This contradicts $\alpha=1 / 2$. We conclude that $A^{\prime} C_{i}^{\prime}=C_{i}^{\prime}$ for $1 \leq i \leq 4$.

(5) We have shown that $A^{\prime} C_{i}^{\prime}=C_{i}^{\prime}$ for all $i$. Since $A^{\prime}$ is orthogonal the first two of these relations show that $A^{\prime}=\operatorname{block} \operatorname{diag}\left(A_{11}, A_{22}\right)$. Thus all symmetries are block diagonal and we may assume that $\alpha=1$, i.e., the symmetry group of $\nu$ and hence $\phi$ is a subgroup of the orthogonal group consisting of block diagonal matrices (in the standard basis).

(6) Let $A \in \mathcal{S}(\nu)=\mathcal{S}(\phi)$ be arbitrary. We now choose a $\theta$ so that the matrix $A^{\prime}=g_{\theta} A$ has the property $A^{\prime} v_{1}=v_{1}$. We shall show that $A^{\prime}=I$, and hence that $A=g_{-\theta}$, thus completing our example. By using the fact that $A^{\prime}$ is orthogonal, and the facts that $A^{\prime} v_{3} \in V_{3}, A^{\prime} v_{4} \in V_{4}$, and $A^{\prime} g_{\pi / 2} v_{3} \in V_{3}$, and computing directly as above, this result is obtained. This completes the example.

\section{Applications}

We will say that a full probability measure $\nu$ on $\mathbf{R}^{d}$ is operator $\alpha$-stable provided that $\nu$ is operator stable with some index $B$, all of whose eigenvalues have real part equal to $1 / \alpha$. The spectral decomposition theorem of Meerschaert (1991) reduces the analysis of generalized domains of attraction to the special case of an operator $\alpha$-stable limit. Suppose that $X$ is in the generalized domain of attraction of some arbitrary operator stable law. Then it is always possible to write $X=X^{(1)}+\cdots+X^{(r)}$ where each component belongs to the generalized domain of attraction of some operator $\alpha$-stable law.

Operator $\alpha$-stable laws are directly analogous to the classical $\alpha$-stable laws on $\mathbf{R}^{1}$. We know that $\alpha \in(0,2]$. If $\alpha=2$ then $\nu$ is a nondegenerate normal law on $\mathbf{R}^{d}$. Suppose then that $\alpha \neq 2$ and that $Y$ is a random vector with distribution $\nu$. The absolute moments $E\|Y\|^{\rho}$ are finite whenever $\rho<\frac{1}{\alpha}$ and infinite whenever $\rho \geq \frac{1}{\alpha}$. If $X$ is attracted to $Y$ then $E\|X\|^{\rho}$ is finite whenever $\rho<\frac{1}{\alpha}$, and infinite whenever $\rho>\frac{1}{\alpha}$. The centering constants $b_{n}$ in (1.1) can be omitted when $\alpha<1$, and when $\alpha>1$ we can center to zero expectation. These facts about moments were established by Hudson, Veeh, and Weiner (1988) and the behavior of the centering constants is due to Meerschaert (1992). 
As in the one variable case, the tail functions $P(\|Y\|>t)$ and $P(\|X\|>$ $t)$ tend to zero about as fast as $t^{-\alpha}$, but in the present case we cannot conclude that the tails are regularly varying. Instead we have the slightly weaker condition that the tails are R-O varying with both upper and lower global index equal to $-\alpha$. See Bingham, Goldie, and Teugels (1987) for information about R-O variation, and Meerschaert (1992) for the proof that the tails of both $X$ and $Y$ are R-O varying. The structure results of this paper give sharper growth rates on the tails. Suppose $\nu$ is operator $\alpha$-stable with commuting exponent $B$. Since $\nu^{t}$ differs from $t^{B} \nu$ by a translation, $B$ governs the tail behavior of $\nu$. Specifically we have from Meerschaert (1990) that $P(|<Y, \theta\rangle \mid>t)=\mathcal{O}\left(1 / t^{*}(r)\right)$ where $t^{*}(r)$ is the asymptotic inverse of $R^{*}(t)=\left\|t^{B^{*}} \theta\right\|$. The asymptotic behavior of $t^{B}$ is governed by the nilpotent operator $N$. Referring to theorem three, we have $\left\|t^{B^{*}} \theta\right\|=\mathcal{O}\left(t^{\frac{1}{\alpha}}(\log t)^{j-1}\right)$ for every unit vector $\theta \in V_{j}^{*}$. For $\theta \in V_{1}^{*}$ we have $P(|\langle Y, \theta\rangle|\rangle$ $t)=\mathcal{O}\left(t^{-\alpha}\right)$, and for all other unit vectors $\theta$, the tails tend to zero more slowly than $t^{-\alpha}$. The fact that $N$ governs the tail behavior of the limit also provides a geometrical interpretation for some of our structure results. Since $N$ determines the decay rate of the tails of $\nu$, it cannot vary with choice of exponent. Since symmetries cannot alter the tail behavior, they must also preserve the direct sum decomposition $\mathbf{R}^{d}=V_{1} \oplus \ldots \oplus V_{n}$ induced by $N$.

As in the one variable case, the norming operators shrink to zero about as fast as $n^{-\frac{1}{\alpha}}$. From theorem 4.1 in Meerschaert (1991) it is not difficult to infer that $\left\|A_{n} \theta\right\|$ is $\mathrm{R}-\mathrm{O}$ varying with both upper and lower global indices equal to $-\frac{1}{\alpha}$ for every unit vector $\theta$. The regular variation condition (1.4) shows that the behavior of norming operators is governed by the exponents and symmetries of the limit law $\nu$. The asymptotic behavior of $A_{n}$ is approximately the same as that of $n^{-B} G_{n}$ where $B$ is an exponent of $\nu$ and $G_{n} \in \mathcal{S}(\nu)$. We can use our structure results to obtain precise information about the behavior of operators of this form. Use the remark 2 following theorem four to write $B=\left(\frac{1}{\alpha}\right) I+Q+N$ where $Q$ is skew symmetric and commutes with $N$. Then $n^{B} G_{n}=n^{\frac{1}{\alpha}} n^{N}\left(n^{Q} G_{n}\right)$. Since $Q$ is skew symmetric, $n^{Q} G_{n}$ is orthogonal. Therefore we have $\left\|n^{B} G_{n} \theta\right\|=\mathcal{O}\left(n^{\frac{1}{\alpha}}(\log n)^{j-1}\right)$ for every unit vector $\theta \in V_{j}$. Note also that $n^{-B} G_{n}$ preserves the spaces $K_{j}=\operatorname{ker} N^{j}$. The authors are currently investigating the extent to which the norming operators $A_{n}$ inherit this behavior.

\section{Acknowledgements.}

In March 1991 Meerschaert was invited to give a talk at Wayne State University in Detroit by Professor Z. Jurek. After the talk Jurek showed Meerschaert some notes written by Veeh in 1986, containing proofs of some of the same results Meerschaert had just finished presenting. Further discussions between Veeh and Meerschaert led to the collaboration which produced this paper. The authors would like to thank Professor Jurek for his help. Thanks also to Professor Wasserman at the University of Michigan for some helpful discussions during the early stages of this research. 


\section{References}

Billingsley, P. (1966) Convergence of types in $k$-space, Z. Wahrsch. verw. Geb. 5, 175-179.

Bingham, N., C. Goldie, and J. Teugels (1987) Regular Variation, Encyclopedia of Mathematics and its Applications, 27, Cambridge University Press, London.

Feller, W. (1971) An Introduction to Probability Theory and Its Applications, vol. 2, 2nd ed., Wiley, New York.

Hirsch, M. and S. Smale (1974) Differential Equations, Dynamical Systems, and Linear Algebra, Academic Press, New York.

Hoffman, K. and Kunze, R. (1961) Linear Algebra, Prentice Hall, New Jersey.

Holmes, J. W. Hudson and J.D. Mason (1982) Operator stable laws: multiple exponents and elliptical symmetry, Ann. Probab. 10, 602-612.

Hudson, W., Z. Jurek and J. Veeh (1986) The symmetry group and exponents of operator stable probability measures, Ann. Probab. 14, 1014-1023.

Hudson, W. , J. Veeh, and D. Weiner (1988) Moments of distributions attracted to operator stable laws, J. Multivariate Anal., 24, 1-10.

Meerschaert, M. (1990) Moments of random vectors which belong to some domain of normal attraction, Ann. Probab. 18, 870-876.

Meerschaert, M. (1991) Spectral decomposition for generalized domains of attraction, Ann. Probab., 19, 875-892.

Meerschaert, M. (1992) Regular variation and generalized domains of attraction in $\mathbf{R}^{k}$, preprint.

Michaliček, J. (1972) Der Anziehungsbereich von Operator-Stabilen Verteilungen $\operatorname{im} R_{2}, Z$. Wahrsch. Verw. Geb. 25 57-70.

Sharpe, M. (1969) Operator stable probability distributions on vector groups, Trans. Amer. Math. Soc. 136, 51-65. 